\section{Prevention of Mother to Child Transmission of Hepatitis B Infection-Need for Holistic Approach}

Apart from the strategies highlighted for prevention of mother to child transmission of hepatitis B infection [1], following measures also need attention.

1. Universal screening for all pregnant women: Screening should be done early in the pregnancy so that those having the infection can be provided appropriate treatment and hence prevent vertical transmission [2].

2. Special focus on high risk pregnant women: Drug addicts, HIV positive and women having other sexually transmitted diseases have higher chances of having hepatitis B co-infection [3]. These women should be provided with regular counselling sessions on the risk of transmission, regular follow up, free treatment and timely referral.

3. Promoting maximum in-hospital deliveries: To identify hepatitis B infected cases and ensure safe delivery practices along with reduction in perinatal complications.

4. Promoting hepatitis B education and universal immunization.
5. Providing free treatment to hepatitis B-infected pregnant women.

6. Updating knowledge of healthcare workers: The doctors working in the rural and remote areas can be updated on the advances in the treatment and prevention of hepatitis B infection by conducting regular Continuing Medical Education programs, and e-learning programs.

7. Free availability of Hepatitis B Immunoglobulin (HBIg): Government should take the initiative to provide free HBIg at all the delivery centers, atleast starting from areas with high prevalence, and then to other parts of the country.

VIKRAM KUMAR
Department of Pediatrics,
Fortis Memorial Research Institute,
Sector-44, Gurgaon, Haryana, India.
viku677@gmail.com

\section{REFERENCES}

1. Geeta MG, Riyaz A. Prevention of mother to child transmission of Hepatitis B infection. Indian Pediatr. 2013;50:189-92.

2. Screening for Hepatitis B Virus Infection in Pregnancy: US Preventive Services Task Force Reaffirmation Recommendation Statement. Ann Intern Med. 2009;150:869-73.

3. Uneke CJ, Ogbu O, Inyama PU, Anyanwu GI, Njoku MO, Idoko JH. Prevalence of hepatitis-B surface antigen among blood donors and human immunodeficiency virus-infected patients in Jos, Nigeria. Mem Inst Oswaldo Cruz. 2005;100:13-6.

\section{Behçet Disease Presenting as Deep Vein Thrombosis and Epididymoorchitis}

Behçet's Disease is a chronic, multisystemic, inflammatory disorder characterized by intraocular inflammation, oral and mucosal ulcerations, skin lesions, and a variety of other manifestations [1]. It occurs rarely in children [2]. A 15-year-old boy was admitted with the complaints of pain in right calf and right testis for 3 days. He had past history of three episodes of high grade fever with nonspecific muscular pain and testicular pain in last one year. He also had a history of recurrent aphthous oral ulcerations that occurred three-to-four times per year accompanied by high fever. On examination, oral apthous ulcers were present, an ulcer was present on right side of the scrotum and his right testis and epidydimis were swollen and tender. Further examination revealed old healed lesions of erythema nodosum over left leg. His right calf muscle was swollen and tender and Homan's sign was positive. Eye examination revealed anterior uveitis.

Hemoglobin, leukocyte count and platelet count were $12.6 \mathrm{~g} / \mathrm{dL}, 15,200 / \mathrm{mm}^{3}$ and $3,50,000 / \mathrm{mm}^{3}$, respectively. C-reactive protein level and erythrocyte sedimentation rate were $7.0 \mathrm{mg} / \mathrm{dL}$ and $93 \mathrm{~mm} /$ hour, respectively. He had no history of sexual exposure and his HIV and VDRL both were negative. Doppler ultrasound of the right leg showed popliteal vein thrombosis. Subcutaneous low 